\title{
Editorial: changes at J-DEDS
}

\author{
Xi-Ren Cao • Stéphane Lafortune
}

Published online: 5 March 2015

(C) 2015 Springer Science+Business Media New York

\section{Farewell:}

The area of discrete event systems (DES) was established in early 1980s; after more than three decades, it has developed into a mature area. This is evidenced by the healthy number of submissions and citations to our Journal. In the past few years, the number of yearly submissions has averaged about 65 and the number of yearly citations has been in the range of 260-292.

Because of its special features, the DES area, as well as this Journal, is facing a number of challenges and opportunities. First, the area of DES deals with theories and applications of systems that are emerging in the new era of information technology; therefore, there is always a need to expand our arena and to accommodate newly emerging subjects. Second, as the founding Editor-in-Chief Yu-Chi (Larry) Ho put it in the inaugural editorial, the key to the answer to the question "Is the baby (journal) ... going to be stunted in its development?" is: "Not if we maintain a problem-driven approach." The developments in the past 24 years provide the best answer to this question. However, the challenge is always there. The area of DES, or that of control and optimization in general, is facing the challenge that it does not "own" a problem domain; problem domains such as manufacturing, communication, networks, logistics, sensor networks, management, discrete event simulation, etc., have their own communities.

In the past decade, we have maintained a close relationship with a number of major conferences in the DES or related areas, including WODES (International Workshop on Discrete Event Systems), ADHS (Analysis and Design of Hybrid Systems), MSR (Modeling of Reactive Systems), and other related conferences in computer science and operations research. This has allowed us to identify newly emerging topics at the frontier of DESrelated research and publish several special issues on topics such as hybrid systems, eventbased control and optimization, performance evaluation methodologies and tools, optimization of DES, and so forth. We also have created the new category of "short papers" for rapid publication of novel but perhaps not fully developed ideas. We are happy to see that many

\footnotetext{
X.-R. Cao $(\bowtie)$

Shanghai Jiaotong University, Shanghai, China

e-mail: eecao@ust.hk

X.-R. Cao

Hong Kong University of Science \& Technology, Hong Kong, China
}

S. Lafortune

The University of Michigan, Ann Arbor, USA 
DES researchers use the journal as one of their major venues to report their new research outcomes.

We also maintain a highly responsible and top quality editorial board and reviewer community. This strong support from authors and reviewers is crucial to ensure high quality. We are also grateful for the excellent and professional support from Springer's editorial office.

After so many years of enjoyable time serving this Journal, I have decided to step down as Editor-in-Chief after nine years in that position. I am delighted that one of the leaders in our field, who has been active in our community for many years, Stéphane Lafortune of the University of Michigan, has kindly agreed to be the new Editor-in-Chief, starting with this first issue of 2015. I truly believe that under his new leadership and with the support from our research community, we will overcome future challenges and J-DEDS will thrive and continue its excellent service to our research community.

\section{Xi-Ren Cao}

\section{Moving Forward:}

I am pleased to accept the position of Editor-in-Chief of J-DEDS. On behalf of the entire DES community, I want to sincerely thank Xi-Ren Cao for his outstanding leadership of our Journal for the past nine years. J-DEDS has a healthy backlog of papers and the quality and diversity of the papers are excellent. I am fortunate to take over with such a good set of "initial conditions" and I will do my best to ensure the continued success of J-DEDS.

I would like to report on some changes to our Editorial and Advisory Boards. Some members have resigned due to other editorial duties or workload constraints. New members have also been added. The changes are detailed below.

Department Editors: Alessandro Giua and Yorai Wardi, who were Associate Editors, will now serve as Department Editors. Les Servi has stepped down.

Associate Editors: The following new Associate Editors have joined the Editorial Board: Jan Komenda, Spyros Reveliotis, and Carla Seatzu. The following members have resigned: Magnus Egerstedt, Stefan Kowalewski, Petar Momcilovic, Meera Sampath, and Leyuan Shi.

Advisory Board: Xi-Ren Cao is joining our Advisory Board. On the other hand, Hanfu Chen and Geer Jan Olsder have stepped down.

I would like to express our gratitude to Les, Magnus, Stefan, Petar, Meera, Leyuan, Hanfu, and Geert Jan for their dedicated service and sound advice the last several years. I would like to welcome Carla, Jan, and Spyros to our Editorial Board; their expertise and considerable editorial experience will be highly beneficial to J-DEDS. Finally, I want to thank Alessandro and Yorai for agreeing to assume the duties of Department Editors, and Xi-Ren for agreeing to continue his involvement with J-DEDS by sharing his wisdom on our Advisory Board.

I am eager to work with the Editorial and Advisory Boards, as well as with the entire research community in DES, to ensure the continued success of our Journal, founded in 1990 by Yu-Chi Ho. J-DEDS has played a key role in establishing the area of discrete event systems as a technological domain at the inferface of systems and control theory, computer science, and operations research. New research challenges abound and I look forward to receiving your submissions on research involving "discrete events" in dynamic systems, from modeling to analysis, control, and optimization, from theory to applications, and from the "expected" to the "unexpected."

Stéphane Lafortune 